\title{
The Relationship of Hyaluronic Acid-CD44 on Cancer Progression and Hyaluronic Acid-Based Self- Assembled Nanoparticle Roles
}

\author{
Mediha CANBEK ${ }^{* 1}$ Ayşe OZMEN YAYLACI ${ }^{1,2}$ and Neda HAMID ${ }^{1}$ \\ ${ }^{1}$ Department of Biology, Faculty of Science, Eskisehir Osmangazi University, 26480 Eskisehir, Turkey \\ ${ }^{2}$ Department of Biology, Faculty of Science, Hitit University, 19030 Corum, Turkey
}

*Corresponding author: Mediha CANBEK, Department of Biology, Faculty of Arts and Science, Eskisehir Osmangazi University,

Campus of Meselik 26480, Eskisehir, Turkey

\section{ARTICLE INFO}

Received: 䊝 October 17, 2019

Published: October 24, 2019

Citation: Mediha CANBEK, Ayșe OZMEN YAYLACI, Neda HAMID. The Relationship of Hyaluronic Acid-CD44 on Cancer Progression and Hyaluronic Acid-Based Self-Assembled Nanoparticle Roles. Biomed J Sci \& Tech Res 22(2)-2019. BJSTR. MS.ID.003730.

Abbreviations: ECM: Extracellular Matrix; HA: Hyaluronic Acid; LMW: Low Molecular Weight HA; RTKs: Receptor Tyrosine Kinases; PDGFR: Platelet-Derived Growth Factor Receptor; RHMAM: Receptor for HA-Mediated Motility; EGFR: Epidermal Growth Factor Receptor
ABSTRACT

Cancer cells have the ability to multiply uncontrollably and metastasize. For metastasizing, they need to change the extracellular matrix (ECM) structure. One of the most important main components of the extracellular matrix is hyaluronic acid (HA). HA fragments and the HA receptor CD44 are very high in cancer cells. The HACD44 receptor relationship in cancer cells is one of the mechanisms that are used to target nanoparticles. Self-assembled nanoparticles are self-organizing nanoparticles. Anticancer drugs that have low solubility in water are transformed with self-assembled nanoparticles and provided with a hydrophilic structure and targeted towards the CD44 receptors that are highly expressed in cancer cells. Anti-cancer drugs of self-assembled HA nanoparticle form can stay in circulation without being destroyed for a long time with surfactant or polymer structures. Additionally, self-assembled HA nanoparticles are more effective on cancer cells and less cytotoxicity on healthy cells. The lifespan quality of cancer patients may be increased by usage of nanoparticles such as target-oriented self-assembled HA nanoparticles.

Keywords: Cancer; Hyaluronic Acid; CD44; Self-Assembled Nanoparticles

\section{Mini Review}

The incidence and mortality rate of cancer are increasing fast worldwide. According to the cancer statistics of the year 2018, it was reported that 18.1 million new cancer cases occurred in the world, and 9.6 million people died of cancer [1]. This number is increasingly higher every year [2]. Cancer cells are cells that multiply uncontrollably. Cancer cells that divide and multiply rapidly may spread to the entire body by invading normal tissues and organs. It is believed that anomalies in multicellular systems such as mutation, chromosomal translocation and deletion accumulate and lead to the disruption of mechanisms that control cell multiplication [2]. Metastatic spreading of cancer cells is a $90 \%$ cause of mortality [3]. In order to eliminate invasion and metastasis in the process of cancer formation, it is needed to understand the behaviors of cancer cells and the nature of their microenvironment. One of the most significant characteristics of cancer cells is that they have the ability to change the extracellular matrix (ECM) to be able to metastasize [3-5]. HA is the main component of the extracellular matrix [6].

Hyaluronic acid (HA) is a mucopolysaccharide with a hydrophilic structure that is found in the interstitial region seen in animal tissues [7]. HA is a long glycosaminoglycan that consists of repeated chains of ( $\beta 1-4)$-glucuronic acid (GlcUA) and ( $\beta 1-3)$ $\mathrm{N}$-acetyl glucosamine (GlcNAc) disaccharide (GAG) and has a high molecular weight (1-10 million $\mathrm{Da}$ ) [8]. HA is one of the main structures of the extracellular matrix and a significant component of connective tissue. Recent studies have shown that, in addition to 
its status as a main structural component, hyaluronic acid is also effective on several cellular functions. It plays a role in important cellular activities such as cell differentiation, growth, cell adhesion, cell migration and proliferation [6].

It is known that low molecular weight HA (LMW-HA) accumulates in various types of cancer. LMW-HA has been associated with various cellular processes such as proliferation, invasion and angiogenesis [9]. Progressive weight reduction of HA fragments can decrease ECM density and cancer cell invasion get easier to other tissues. In addition to this, inflammation, angiogenesis, metastasis and multidrug resistance (MDR) increase [10]. While LMW-HA contributes to favoring cancer cells, HMW-HA has the opposite effect [11], just as in its protective effect against cancer in naked mole rats.

Naked mole rats have a much longer lifespan in comparison to other rodents. While the average lifespan of mice and other rodents is about 4 years, this time is 32 years for naked mole rats. Several scientists reported that HA, provides animals resistance to cancer [12]. The reason for these rats to not get cancer is the HMW-HA, they possess which is approximately 5 times larger than that in mice and humans. This special HMW-HA is synthesized by fibroblasts forms a tight density around the cells. It is believed that this cloud of HMWHA that surrounds cells prevents not only transformation of normal cells into cancer cells but also metastasis. The thing which makes this hyaluronic acid special is the structural differentiation of HAS2 synthase, which is one of the enzymes that synthesize hyaluronic acid, in difference to the case in humans $[13,14]$. Such a large HMWHA cannot be synthesized in humans [9].

In addition to the presence of HMW-HA, prevention of cancer formation in naked mole rats also involves the mechanisms of p16 induction (HMW-HA mediated) and early contact inhibition [14-16]. HA is different to other GAGs. HA is not synthesized from the Golgi apparatus as a single protein such as proteoglycan. It is synthesized by mediation of the HA synthases that are found in the inner surface of the plasma membrane (HAS1, HAS2, HAS3). While HAS1 and HAS2 synthesize HA with high molecular weights, HAS3 is responsible for synthesis of HA with low molecular weights [8]. There are six hyaluronidases responsible for degradation of hyaluronic acid (Hyal-1, Hyal-2, Hyal-3, Hyal-4, PH-20) Sugahara et al. Among these, Hyal-1 and Hyal-2 have been studied the most. Different molecular weights of hyaluronic acid play a role in formation of different cellular signals [8]. There are two main HA receptors: Cluster of differentiation (CD44) and Receptor for HAMediated Motility (RHAMM) [8]. Besides these, it is known that both HMW-HA and LMW-HA bond with the cell surface receptors of Toll Like Receptors-2 (TLR-2), TLR-4, Lymphatic Vessel Hyaluronan Receptor (LYVE) and layilin and some ECM molecules such as collagen VI and Proteoglycans $[4,11,17]$. As the receptor CD44 has appeared to be associated with cancer more in comparison to other receptors, it has been researched in more detail.

CD44 is found in endothelial cells, leukocytes, epithelial cells, fibroblasts, keratinocytes, hematopoietic cells and various cancer cells $[8,18]$. It has a glycoprotein structure, and it passes the membrane along its axis. It has 7 extracellular domains, a transmembrane domain and a cytoplasmic domain. While the N-terminal is found in the extracellular region, the C-terminal is inside the cell. There is a "link module" motif in the N-terminal where HA and HA-bonded proteoglycans can bond [8]. The end of the C-terminal is associated with cytoskeleton proteins [2]. CD44 has great significance for cell adhesion during the cell migration and tissue formation [2]. The adhesion and spreading activities of lymphocytes are also associated with CD44 [19]. It plays a role in extracellular vesicle interaction [20]. Besides these, it is known to play a role in physiological processes such as hematopoiesis and extremity development [20].

CD44 has several isoforms that are formed by alternative splicing of one gene in the 20-exon region. This diversity increases by post-translational modifications ( $\mathrm{N}$ and 0-glycosylation) [2]. CD44 receptors that are formed by alternative splicing taking place in the 6-15 exons of the CD44 gene are known as "variants" (CD44v) [21]. The standard CD44 isoform is CD44s [2]. CD44v is expressed in extraordinary circumstances such as development and tumors. Among these, high expression of CD44v6 in different types of cancer has been reported, and it is known that it is effective in cancer formation and progression $[8,20]$. Hyaluronic acid is not the only ligand of the CD44 receptor, and CD44 gains the ability to bond with different ligands by exposure to post-translational modifications. These include collagen, fibronectin, laminin, fibrin, osteopontin (OPN) and serglycin [11].

CD44 isoforms affect some receptors on the cell surface and regulate their functions. These receptors include those that play a role on cancer such as Receptor Tyrosine Kinases (RTKs) like c-Met, the Vascular Endothelial Growth Factor Receptor-2 (VEGFR-2), Platelet-Derived Growth Factor Receptor (PDGFR) and Epidermal Growth Factor Receptor (EGFR). These are effective on activation and inhibition of several signal pathways such as Wnt and Fas. By affecting the functions of such pathways and receptors, they have significant effects on inhibition of apoptosis mechanisms in cancer cells and development of drug resistance $[8,11]$.

It was shown that HA, HA synthase, Hyaluronidases and hyaluronic acid receptors increase in various types of cancer [22]. However, their mechanisms in the cancer formation process have not been enlightened yet $[5,22]$. Nevertheless, some possible mechanisms related to this issue have been proposed. The first one of these was associated with the Warburg effect that is seen in cancer cells [11]. Accordingly, cancer cells prefer the way of anaerobic respiration to be able to produce ATP and require too much glucose to be able to produce sufficient energy. This hyperglycemic environment created by cancer cells by excessive glucose intake leads to an increase in, UDP-N-acetylglucosamine. This situation triggers dimerization and expression of HAS2, which is an HA synthase. The increase in HAS2 quantities leads to an increase in the quantity of HA, and an HA cloud is formed around cancer cells. Through deg- 
radation of HA by hyaluronidases, LMW-HA is formed. LMW-HA, by bonding with $\mathrm{CD} 44$ receptors, contributes to processes of invasion, neoangiogenesis and metastasis. Moreover, it was reported at CD44v6 shows an anti-apoptotic effect as a result of its collaboration with receptor tyrosine kinases, contributes to drug resistance and affects HA synthesis in an increasing direction [11].

The HA-CD44 ligand/receptor relationship is utilized nowadays to design nanoparticles that are used for cancer treatment. Anticancer drug are used together with carrier nanoparticles, the time they spend in circulation increases, and they directly target cancer cells. This is why they become more effective in lower doses in comparison to the dose used in chemotherapy, and they affect healthy cells to a minimal extent, some side effects that are seen in chemotherapy patients are eliminated/reduced. This increases the quality of life of cancer patients [2,23]. There are various HA-based nanomaterials that are used for the purpose of cancer treatment. These include polymeric drug-conjugated HA, nanomaterials (such as: micelles, polymersome, hydrogels)and inorganic nanoparticle materials [24]. One example of these may be self-assemble nanoparticles. Self-assembled HA nanoparticles are drug carriers that are frequently utilized in cancer treatment, because the expression of the HA receptor CD44 in cancer cells is very high [25].

Self-assembled nanoparticles consist of three parts. These are the surface, shell and core [26]. Such nanoparticles have a hydrophobic core and a hydrophilic shell. The hydrophobic core serves collection of drugs with a hydrophobic structure. The outer surface of the hydrophilic shell has surfactants, and these constitute the surface part of the nanoparticle $[23,26]$. These molecules found in the nanoparticle surface are monofunctional long-chain molecules such as amines, phosphines, carboxylates and thiols. These molecules provide nanoparticle stability. Among these, the most frequently preferred one is Polyethylene glycol (PEG) [26]. As nanoparticles with a PEG structure are not noticed by phagocytic cells, they have a lower rate of opsonization, and they show a more effective accumulation in cancerous tissues [27].

Self-assembled nanoparticles show a self-forming behavior in aqueous environments due to their amphiphilic structures. Anticancer drugs that have low solubility in water are transformed with self-assembled nanoparticles and provided with a hydrophilic structure. Various polymers and surfactants are used in the surface part of nanoparticles [26]. These provide the nanoparticle with a surface charge. In addition to this, they passively play a role in carrying the nanoparticle to the targeted cancer cells [28] and allow it to stay in circulation [26]. Additionally, structures such as antibodies, receptors, small organic molecules that are added to the surface parts of nanoparticles play a role in recognition of nanoparticles by tumor tissues, as well as their bonding and intake into the cell $[27,29]$. One of such molecules is HA, which is used to target nanoparticles towards cancer cells that are known to show high expression of CD44 [30,31]. The surfaces of cancer cells are highly rich in terms of CD44 receptors. Self-assembled HA nanoparticles that effectively access the tumor tissue are bonded with the CD44 receptors that are found in abundance on the surfaces of cancer cells.

The Hyal-2 that is found on the cell surface starts degradation of the nanoparticle structure by degrading the HA molecules in this structure. Self-assembled-HA nanoparticles are taken into the cancer cells by CD44 receptor-mediated endocytosis.The partly degraded nanoparticles are degraded even more by the Hyal-1 inside the cell, and the anticancer agent is dispersed within the cell [32]. There are various cancer studies that have been carried out on self-assembled HA nanoparticles. The study by Choi et al. examined the properties of self-assembled HA nanoparticles and revealed that HA nanoparticles have a high ability of being targeted towards cancer cells [25]. Choi et al. introduced self-assembled HA nanoparticles with an without PEG and it was reported that PEG-coated self-assembled HA nanoparticles stayed in circulation for a longer time, and they showed higher accumulation in the tumor tissue in comparison to the HA nanoparticles without PEG coating [33].

In a similar study, PEG was added to the structure of selfassembled HA nanoparticles to be used in carrying the anticancer agents, doxorubicin and camptothecin, to cancer tissues. It was shown that the nanoparticle forms were more effective in comparison to those that were freely provided [32]. In the study by Huang et al. (2013), docetaxel-PLGA-HA nanoparticles showed an anti-tumor effect by reducing systemic toxicity in breast cancer mice [23]. Self-assembled HA nanoparticles that were used in another study were proven to block CD44 receptors in colon cancer cells [31]. In the study by Ganesh et al. [34], siRNA trapped in selfassembled nanoparticles inhibited the high expression of CD44 [34]. Self-assembled HA nanoparticles may be easily targeted towards cancer cells with high CD44 expression. Due to the surfactants or polymers added to the nanoparticle surfaces such as PEG, they can stay in circulation for a long time without opsonization by the reticuloendothelial system, and they show a more effective cytotoxic influence on cancer cells. The lifespan and quality of life of cancer patients may be increased by usage of nanoparticles such as target-oriented self-assembled HA nanoparticles.

\section{Acknowledgement}

None.

\section{Conflict of Interest}

No conflict of interest.

\section{References}

1. Bray F, Ferlay J, Soerjomataram I, Siegel RL, Torre LA, et al. (2018) Global cancer statistics 2018: Globocan estimates of incidence and mortality worldwide for 36 cancers in 185 countries. CA: A Cancer Journal for Clinicians 68(6): 394-424.

2. Mattheolabakis G, Milane L, Singh A, Amiji MM (2015) Hyaluronic acid targeting of CD44 for cancer therapy: From receptor biology to nanomedicine. Journal of Drug Targeting 23(7-8): 605-618. 
3. Canel M, Serrels A, Frame MC, Brunton VG (2013) E-cadherin integrin crosstalk in cancer invasion and metastasis. Journal of Cell Science Journal of Cell Science Zaidel-Bar J Cell Sci 126(Pt2): 393-401.

4. Karousou E, D Angelo ML, Kouvidi K, Vigetti D, Viola M, et al. (2014) Collagen VI and hyaluronan: the common role in breast cancer. BioMed research international 606458.

5. Dosio F, Arpicco S, Stella B, Fattal E (2016) Hyaluronic acid for anticancer drug and nucleic acid delivery. Advanced Drug Delivery Reviews 97: 204-236.

6. Collins MN, Birkinshaw C (2013) Hyaluronic acid based scaffolds for tissue engineering-A review. Carbohydrate Polymers 92(2): 1262-1279.

7. Meyer K (1947) The Biological Significance of Hyaluronic Acid and Hyaluronidase. Physiological reviews 3: 335.

8. Misra S, Hascall VC, Markwald RR, Ghatak S (2015). Interactions between hyaluronan and its receptors (CD44, RHAMM) regulate the activities of inflammation and cancer. Frontiers in Immunologymunolog 6: 201.

9. Mima K, Beppu T, Ishiko T, Chikamoto A, Nakagawa S, et al. (2014) Preoperative serum hyaluronic acid level as a prognostic factor in patients undergoing hepatic resection for hepatocellular carcinoma. British Journal of Surgery 101(3): 269-276.

10. Dosio F, Arpicco S, Stella B, Fattal E (2016) Hyaluronic acid for anticancer drug and nucleic acid delivery. Advanced Drug Delivery Reviews 97: 204-236.

11. Karousou E, Misra S, Ghatak S, Dobra K, Götte M, et al. (2016) Roles and targeting of the HAS/ hyaluronan/CD44 molecular system in cancer 59:3-22.

12. Ecol M (2014) Comparative genetics of longevity and cancer: insights from long-lived rodents. Nat Rev Genet 15(8): 531-540.

13. Fisher GJ (2015) Cancer resistance, high molecular weight hyaluronic acid, and longevity. Journal of Cell Communication and Signaling 9(1): 91-92.

14. Tian X, Azpurua J, Hine C, Vaidya A, Myakishev Rempel M, et al. (2013). High molecular weight hyaluronan mediates the cancer resistance of the naked mole-rat 499(7458): 346-349.

15. Schuhmacher, Zoé Husson, Ewan St John Smith LN (2015) The naked mole-rat as an animal model in biomedical research: current perspectives. Open Access Animal Physiology 7: 137-148.

16. Tian X, Azpurua J, Ke Z, Augereau A, Zhang ZD, et al. (2014) INK4 locus of the tumor-resistant rodent, the naked mole rat, expresses a functional p15/p16 hybrid isoform. PNAS 112(4): 1053-1058.

17. Bono P, Cordero E, Johnson K, Borowsky M, Ramesh V, et al. (2005) Layilin, a cell surface hyaluronan receptor, interacts with merlin and radixin. Experimental Cell Research, 308(1): 177-187.

18. Ghezzi P, Naor D (2016) Editorial: interaction Between Hyaluronic acid and its receptors (Cd44, rHaMM) regulates the activity of inflammation and Cancer The Editorial on the Research Topic Interaction Between Hyaluronic Acid and Its Receptors (CD44, RHAMM) Regulates the Activity 7.

19. Föger N, Marhaba R, Zöller M (2001) Involvement of CD44 in cytoskeleton rearrangement and raft reorganization in T cells. Journal of Cell Science 114(6): 1169-1178.
20. Kai Härkönen, Pyysalo S, Hakkola S, Ketola K, Oliveira C, et al. (2018) OF13.05 ISEV 2018 abstract book 103 Exosomal heparan sulphate proteoglycans (HSPGs) drive induction of a pro-angiogenic stromal cell phenotype Alex P. Shephard; Zsuzsanna Tabi; Rachel Errington; Aled Clayton; Jason P. Webber Tissue Microenvironment Group, ISEV 2018 abstract book, OF13.06: 103.

21. Senbanjo LT, Chellaiah MA (2017) CD44: A multifunctional cell surface adhesion receptor is a regulator of progression and metastasis of cancer cells. Frontiers in Cell and Developmental Biology 5: 18

22. Sironen RK, Tammi M, Tammi R, Auvinen PK, Anttila M, et al. (2011) Hyaluronan in human malignancies. Experimental Cell Research 317(4): 383-391.

23. Huang J, Zhang H, Yu Y, Chen Y, Wang D, et al. (2014). Biodegradable selfassembled nanoparticles of poly (d,l-lactide-co-glycolide)/hyaluronic acid block copolymers for target delivery of docetaxel to breast cancer. Biomaterials 35(1): 550-566.

24. Kim JH, Moon MJ, Kim DY, Heo SH, Jeong YY (2018) Hyaluronic acidbased nanomaterials for cancer therapy. Polymers 10(10): 1-15.

25. Choi KY, Min KH, Na JH, Choi K, Kim K, et al. (2009) Self-assembled hyaluronic acid nanoparticles as a potential drug carrier for cancer therapy: synthesis, characterization, and in vivo biodistribution. Journal of Materials Chemistry 19(24): 4102-4107.

26. Christian P, Von der Kammer F, Baalousha M, Hofmann T (2008) Nanoparticles: structure, properties, preparation and behaviour in environmental media. Ecotoxicology 17(5): 326-343.

27. Sun T, Zhang YS, Pang B, Hyun DC, Yang M, et al. (2014) Engineered nanoparticles for drug delivery in cancer therapy. Angewandte Chemie International Edition 53(46): 12320-12364.

28. Nagarajan R, Ruckenstein E (1991) Theory of Surfactant Self-Assembly: A Predictive Molecular Thermodynamic Approach. Langmuir 7(12): 2934-2969.

29. Choi KY, Chung H, Min KH, Yoon HY, Kim K, et al. (2010) Self-assembled hyaluronic acid nanoparticles for active tumor targeting 31(1): 106-114.

30. Cho HJ, Yoon HY, Koo H, Ko SH, Shim JS, et al. (2011) Self-assembled nanoparticles based on hyaluronic acid-ceramide (HA-CE) and Pluronic $\AA$ for tumor-targeted delivery of docetaxel. Biomaterials 32(29): 7181-7190.

31. Jeong YI, Kim DH, Chung CW, Yoo JJ, Choi KH, et al. (2012) Self-assembled nanoparticles of hyaluronic acid/poly(dl-lactide-co-glycolide) block copolymer. Colloids and Surfaces B: Biointerfaces 90: 28-35.

32. Choi KY, Yoon HY, Kim JH, Bae SM, Park RW, et al. (2011) Smart nanocarrier based on PEGylated hyaluronic acid for cancer therapy. ACS Nano 5(11): 8591-8599.

33. Choi KY, Min KH, Yoon HY, Kim K, Park JH, et al. (2011) PEGylation of hyaluronic acid nanoparticles improves tumor targetability in vivo. Biomaterials 32: 1880-1889.

34. Ganesh S, Iyer AK, Morrissey DV, Amiji MM (2013) Hyaluronic acid based self-assembling nanosystems for CD44 target mediated siRNA delivery to solid tumors. Biomaterials 34(13): 3489-3502. 
ISSN: 2574-1241

DOI: 10.26717/BJSTR.2019.22.003730

Mediha CANBEK. Biomed J Sci \& Tech Res

(C) (i) This work is licensed under Creative

Submission Link: https://biomedres.us/submit-manuscript.php

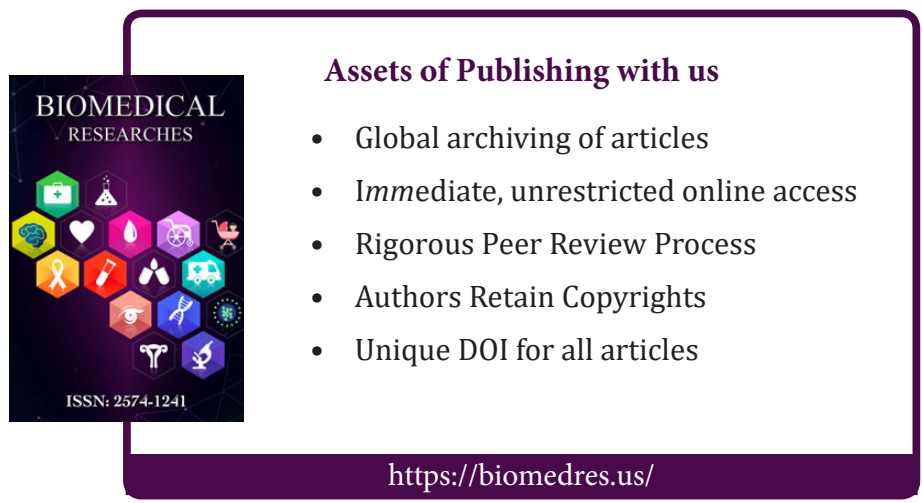

